\title{
The Pluricomplex Green Function, Capacitative Notions, and Approximation Problems in $\mathbf{C}^{n}$
}

\section{Stephanie Nivoche}

ABstract. We prove here the following result: If $D$ is a bounded strictly hyperconvex domain in $\mathbf{C}^{n}$ containing $O$, then the sequence of $\xi$-directional analytic capacities converges to the $\xi$-directional harmonic capacity, quasi-everywhere in $S(O, 1)$. We deduce two results from this property. The first one is a comparison between the $\xi$ directional harmonic capacities associated respectively to the Green function of $\mathbf{C}^{n} \backslash K$ with pole at infinity and to the pluricomplex Green function of the dual open set $K^{*}$ with pole at $O$. The second is a characterization of the harmonic capacity associated to the dual domain $K^{*}$ of a regular Martineau compact $K$ in $\mathbf{C}^{n}$ by the polynomial approximations of the exponential function on $K$.

1. Introduction. Let $D$ be a bounded hyperconvex domain in $\mathbf{C}^{n}$ containing $O$. L. Lempert [10], M. Klimek [7] and J. P. Demailly [3] proved the existence and the uniqueness of a function $g_{D}$, continuous on $\bar{D}$ with values in $[-\infty, 0]$, plurisubharmonic in $D$ such that $\left.g_{D}\right|_{b D} \equiv 0,\left(d d^{c} g_{D}\right)^{n}=0$ on $D \backslash\{O\}$ and $g_{D}(z) \sim \log \|z\|$ when $z \rightarrow O$. This function exists if and only if the bounded domain $D$ is hyperconvex. In this case, this function is called the pluricomplex Green function with logarithmic pole at $O$ in $D$ and it satisfies $\left(d d^{c} g_{D}\right)^{n}=$ $(2 \pi)^{n} \delta_{O}$ and

$$
\begin{aligned}
& g_{D}(z)=\sup \left\{v(z): v \in \operatorname{PSH}(D,[-\infty, 0]), v(y) \leq \log \|y\|+O_{v}(1)\right. \\
&\text { when } y \rightarrow O\} .
\end{aligned}
$$

For $\xi \in S(O, 1)$ (the unit sphere in $\mathbf{C}^{n}$ ), we define the $\xi$-directional harmonic capacity $C_{D}(\xi)$ of the domain $D$ by:

$$
-\log C_{D}(\xi)=\limsup _{\lambda \in \mathbf{C}, \lambda \rightarrow 0}\left(g_{D}(\lambda \xi)-\log |\lambda|\right) .
$$


$C_{D}(\xi)$ was introduced by P. Lelong in [9] and E. Bedford and B. A. Taylor [2] called $\varrho_{D}(\xi)=-\log C_{D}(\xi)$ the Robin function of the domain $D$. In Section 2 , we study the convergence of sequences $\left(g_{D_{j}}\right)$ and $\left(C_{D_{j}}\right)$, where $\left(D_{j}\right)$ is an increasing or decreasing sequence of domains.

Let $D$ be a domain in $\mathbf{C}^{n}$ containing $O$ and $p$ be an integer $\geq 1$. We define a compact set $\mathcal{E}_{p}(D)$ of $H(D)$ (the complex vector space of holomorphic functions in $D$, with the topology of uniform convergence on every compact set of $D$ ) and a continuous and plurisubharmonic function $h_{p}$ on $D$ by:

$$
\begin{aligned}
\mathcal{E}_{p}(D) & =\left\{f \in H(D): \sup _{z \in D}|f(z)| \leq 1, D^{(\nu)} f(O)=0 \forall \nu \in \mathbf{N}^{n} /|\nu| \leq p-1\right\}, \\
h_{p}(z) & =\sup _{f \in \mathcal{E}_{p}(D)} \frac{1}{p} \log |f(z)|, \forall z \in D .
\end{aligned}
$$

For every $\xi \in S(O, 1)$, let $\gamma_{p}(D, \xi)$ be the $\xi$-directional analytic capacity of order $p$ defined by

$$
\gamma_{p}(D, \xi)=\left\{\max \left\{\left|d_{\xi}^{(p)} f(0) / p !\right|^{1 / p}: f \in \mathcal{E}_{p}(D)\right\}\right\}^{-1} .
$$

$h_{p}$ and $\gamma_{p}$ were introduced in [13] for $n=1$, and in [6] and [14] for the multidimensional case.

From a result of L. Lempert [10], it follows that on a strictly convex domain $D$ in $\mathbf{C}^{n}$ with analytic boundary, $h_{p}=g_{D}$ on $D$ and $\gamma_{p}(D, \xi)=C_{D}(\xi)$ on $S(O, 1)$, $\forall p \geq 1$. The main result of this article is the following theorem which generalizes the property above:

Theorem 1.1. If $D$ is a bounded strictly hyperconvex domain in $\mathbf{C}^{n}$ containing $O$, then

$$
\begin{aligned}
\lim _{p \rightarrow \infty} h_{p}(z) & =g_{D}(z) \quad \forall z \in D \\
\lim _{p \rightarrow \infty} \gamma_{p}(D, \xi) & =C_{D}(\xi) \quad \text { quasi-everywhere in } S(O, 1) .
\end{aligned}
$$

From this theorem which is proved in Section 3, we deduce two corollaries. The first one, in Section 4, gives a comparison between several capacities. If $K$ is a non-pluripolar compact set in $\mathbf{C}^{n}$ with the generalized Green function $g_{K}$, for all $\xi \in S(O, 1)$, we define $C_{K}(\xi)$, the $\xi$-directional harmonic capacity of the 
compact set $K$ by:

$$
-\log C_{K}(\xi)=\limsup _{\lambda \in \mathbf{C},|\lambda| \rightarrow \infty}\left(g_{K}(\lambda \xi)-\log |\lambda|\right)
$$

The function $-\log C_{K}(\xi)$ is the Robin function of the compact set $K$ [19]. We also generalize the notion of Tchébycheff constant in the multidimensional case introducing constants $\tau^{*}(K, \xi)$ and $\tau(K, \xi)$ and obtain the following corollary, in which we use definitions from Section 4 .

Theorem 1.2. Let $K$ be a compact set in $\mathbf{C}^{n}$.

(1) If its dual open set $K^{*}$ is a bounded hyperconvex domain in $\mathbf{C}^{n *}$, then we have

$$
C_{K^{*}}(\xi) \leq \tau^{*}(K, \xi)^{-1} \leq \tau(K, \xi)^{-1} \leq C_{K}(\bar{\xi})^{-1}, \quad \forall \xi \in S(O, 1)
$$

(2) If $K$ is L-regular, we have $\tau(K, \xi)=C_{K}(\bar{\xi}), \forall \xi \in S(O, 1)$.

(3) If in addition, $K$ is a regular Martineau compact then we get finally that $C_{K^{*}}(\xi)=\tau^{*}(K, \xi)^{-1}$ quasi-everywhere in $S(O, 1)$.

In the case of a compact set $K$ in $\mathbf{C}$ endowed with a Green function with pole at infinity, we have $g_{K}(z)=-g_{K^{*}}(-1 / z)$ on $\mathbf{C}$ and $C(K)=C\left(K^{*}\right)^{-1}$. Also according to G. Szegö (1924), we have $C(K)=\tau(K)$. In $\mathbf{C}^{n}$, the situation is different. If $K$ is a closed polydisk in $\mathbf{C}^{n}$ with center at $O$, then there exist points $\xi$ in $S(O, 1)$ satisfying $C_{K^{*}}(\xi)<C_{K}(\bar{\xi})^{-1}$.

The second corollary, which is proved in Section 5 , characterizes the $\xi$-directional harmonic capacity of the dual domain $K^{*}$ of a regular Martineau compact $K$, in terms of polynomial approximations of the exponential function on $K$. This corollary generalizes a result of G. M. Henkin and A. Shananin [5]. For $N \in \mathbf{N}$, we denote by $\mathcal{P}_{N}$ the complex vector space of holomorphic polynomials of degree less or equal to $N$. If $K$ is a compact set in $\mathbf{C}^{n}$ and $f$ is a continuous function on $K$, then $E_{N}(K, f)=\inf \left\{\sup \{|f(z)-p(z)|: z \in K\}: p \in \mathcal{P}_{N}\right\}$. If we denote by $\mathcal{C}(K)$ the Banach space of continuous functions on $K$ with the uniform norm on $K$, then $E_{N}(K, f)$ is the distance in $\mathcal{C}(K)$ between the function $f$ and the closed subspace $\mathcal{P}_{N}$.

Theorem 1.3. If $K$ is a regular Martineau compact set in $\mathbf{C}^{n}$ with dual domain $K^{*}$, then for every complex number $\lambda$ and quasi-everywhere in $S(O, 1)$

$$
\lim _{N \rightarrow \infty} N \cdot E_{N}^{1 / N}\left(K, e^{\lambda \xi \cdot z}\right)=e|\lambda| C_{K^{*}}(\xi)^{-1}
$$

The main results of this article were announced in [14]. 
Notations. On $\mathbf{C}^{n}$, we only use the hermitian norm: $\|z\|=\left(\sum_{j=1}^{n}\left|z_{j}\right|^{2}\right)^{1 / 2}$ and we note $z \cdot z^{\prime}=\sum_{j=1}^{n} z_{j} z_{j}^{\prime}$ if $z=\left(z_{1}, \ldots, z_{n}\right)$ and $z^{\prime}=\left(z_{1}^{\prime}, \ldots, z_{n}^{\prime}\right) \in \mathbf{C}^{n}$. Let $R$ be a positive real number. We will denote by $D(0, R)$ the open disk with center at 0 and radius $R$ in $\mathbf{C}$ and by $B(O, R)$ the open ball with center at $O$ and radius $R$ in $\mathbf{C}^{n}$. If $R=\left(R_{1}, \ldots, R_{n}\right) \in\left(\mathbf{R}^{+}\right)^{n}$ and $z \in \mathbf{C}^{n}$, we will denote by $P(z, R)$ the open polydisk with center at $z$ and multiradius $R$. If $f \in \mathcal{C}^{\infty}(D)$ (where $D$ is an open set in $\mathbf{C}^{n}$ ), then for every $\xi \in S(O, 1)$ and all $p \in \mathbf{N}$,

$$
d_{\xi}^{(p)} f(0)=\left.\frac{\partial^{p}(f(\lambda \xi))}{\partial \lambda^{p}}\right|_{\lambda=0}
$$

is the $\xi$-directional derivative of order $p$ of the function $f$ at $O$. We will use the abreviation psh for plurisubharmonic; $\operatorname{PSH}(D)$ is the set of psh functions on the open set $D$ and $H(D)$ is the set of holomorphic functions on $D$.

Acknowledgments. This paper was written when I was in the postgraduate school of Pierre and Marie Curie University. I am very grateful to my thesis advisor Professor G. Henkin for his advice and help. I also would like to thank the referee whose suggestions and corrections significantly improved this paper.

2. Pluricomplex Green function and harmonic capacity. A domain $D$ in $\mathbf{C}^{n}$ is called hyperconvex if there exists a continuous psh exhaustion function $\varrho: D \rightarrow]-\infty, 0[$. This definition has been introduced by J. L. Stehlé. In what follows, we will often need the notion of strictly hyperconvex domain defined as below.

Definition 2.1. A bounded domain $D$ in $\mathbf{C}^{n}$ is said to be strictly hyperconvex if there exists a bounded domain $\Omega$ and a function $\varrho \in \mathcal{C}(\Omega]-,\infty, 1[) \cap$ $\operatorname{PSH}(\Omega)$ such that $D=\{z \in \Omega: \varrho(z)<0\}$, $\varrho$ is exhaustive for $\Omega$ and for all real number $c \in[0,1]$, the open set $\{z \in \Omega: \varrho(z)<c\}$ is connected.

The two following properties study the convergence of sequences $\left(g_{D_{j}}\right)$ and $\left(C_{D j}\right)$, where $\left(D_{j}\right)$ is an increasing or decreasing sequence of hyperconvex domains.

Proposition 2.2. ([3], [2]) Let $D$ be a bounded hyperconvex domain in $\mathbf{C}^{n}$ containing $O$. If $\left(D_{j}\right)$ is an increasing sequence of hyperconvex domains in $\mathbf{C}^{n}$ containing $O$ such that $\bigcup_{j} D_{j}=D$, then the sequence $\left(g_{D_{j}}\right)$ converges pointwise on $D$ to $g_{D}$ and the sequence $\left(C_{D j}(\xi)\right)$ converges on $S(O, 1)$ to $C_{D}(\xi)$.

Proposition 2.3. Let $D$ be a bounded strictly hyperconvex domain in $\mathbf{C}^{n}$ containing $O$ (as in Definition 2.1). If $\left(D_{j}\right)_{j \geq 1}$ is the decreasing sequence of bounded hyperconvex domains $D_{j}$, defined by $D_{j}=\{z \in \Omega: \varrho(z)<1 / j\}$ for 
every $j \geq 1$, then: the sequence $\left(g_{D_{j}}\right)_{j \geq 1}$ converges uniformly to $g_{D}$ on $\bar{D}$

$$
\lim _{j \rightarrow \infty} C_{D_{j}}(\xi)=C_{D}(\xi), \quad \text { for all } \xi \text { in } S(O, 1) .
$$

Proof. First we verify that $\lim _{j} g_{D_{j}}(z)=0=g_{D}(z), \forall z \in b D$. Next, for every $j \geq 1$ let $c_{j}=\inf _{z \in b D} g_{D_{j}}(z)$. Since $g_{D_{j}}$ is a negative, continuous and real valued function on the compact set $\left.b D, c_{j} \in\right]-\infty, 0\left[\right.$. The sequence $\left(g_{D_{j}}\right)$ increases and converges pointwise to the restriction of the function $g_{D}$ on the boundary of $D$, i.e., to 0 . Then according to Dini's theorem, the sequence $\left(g_{D_{j}}\right)$ converges uniformly to 0 on $b D$ and $\lim _{j} c_{j}=0$. For every $j \geq 1$, consider $g_{j}$ the function defined on $D_{j}$ by

$$
g_{j}(z)= \begin{cases}g_{D_{j}}(z) & \text { if } z \in D_{j} \backslash D, \\ \max \left\{g_{D_{j}}(z), g_{D}(z)+c_{j}\right\} & \text { if } z \in \bar{D} .\end{cases}
$$

$g_{j}$ is a negative continuous psh function on $D_{j}$ and it has a logarithmic pole at $O$. Consequently $g_{j} \leq g_{D_{j}}$ on $D_{j}$ and in particular, $g_{D}+c_{j} \leq g_{D_{j}}$ on $\bar{D}$. Also $D \subset D_{j}$ and $g_{D_{j}} \leq g_{D}$. Thus we get the two following statements:

$$
\begin{array}{cl}
g_{D_{j}} \leq g_{D} \leq g_{D_{j}}-c_{j}, & \text { on } \bar{D}, \\
\text { and } \quad C_{D_{j}}(\xi) \geq C_{D}(\xi) \geq C_{D_{j}}(\xi) e^{c_{j}}, & \forall \xi \in S(O, 1) ;
\end{array}
$$

and the proposition is proved.

\section{Analytic capacities of a domain in $\mathbf{C}^{n}$.}

Lemma 3.1. Let $D$ be a domain in $\mathbf{C}^{n}$ containing $O$. Then:

(1) $\forall p \geq 1, h_{p}$ is a continuous and psh function on $D$, with values at $[-\infty, 0[$.

(2) $h_{p}(z)$ is equivalent to $\log \|z\|$ when $z$ tends to $O$.

(3) $h_{p}(\lambda \xi)-\log |\lambda|$ converges to $-\log \gamma_{p}(D, \xi)$ when $\lambda\left(\in \mathbf{C}^{*}\right)$ tends to 0 , $\forall \xi \in S(O, 1)$.

(4) The sequences $\left(h_{p}\right)$ and $\left(\gamma_{p}(D, \xi)\right)$ satisfy: $\forall(p, q) \in\left(\mathbf{N}^{*}\right)^{2}$

$$
\begin{aligned}
(p+q) h_{p+q} & \geq p h_{p}+q h_{q}, \quad \text { on } D \\
\gamma_{p+q}(D, \xi)^{(p+q)} & \leq \gamma_{p}(D, \xi)^{p} \cdot \gamma_{q}(D, \xi)^{q}, \quad \text { on } S(O, 1),
\end{aligned}
$$

and they converge $\forall z \in D$ and $\forall \xi \in S(O, 1)$, respectively. 
Proof. For every $f \in \mathcal{E}_{p}(D),(\log |f|) / p$ is a continuous function on $D$, so $h_{p}$ is lower semicontinuous on $D$. By using Montel's Theorem and the fact that $\mathcal{E}_{p}(D)$ is a compact set in $H(D)$, it follows that $h_{p}$ is also upper semicontinuous on $D$. The plurisubharmonicity of $h_{p}$ is a consequence of its definition.

If $B(O, r) \subset D \subset B(O, R)$, let us show that $\log (\|z\| / R) \leq h_{p}(z) \leq \log (\|z\| / r)$ on $D$. If $z \in D \backslash\{O\}$, then $z=\|z\| \xi_{0}$, where $\xi_{0}=z /\|z\| \in S(O, 1)$. The function $f(w)=\left(w \cdot \bar{\xi}_{0}\right)^{p} / R^{p}$ belongs to $\mathcal{E}_{p}(D)$ and $h_{p}(z) \geq \log (\|z\| / R)$. By Schwarz's Lemma for the ball $B(O, r) \subset D$, we obtain $|f(z)| \leq\|z\|^{p} / r^{p}, \forall z \in B(O, r)$, and $\forall f \in \mathcal{E}_{p}(D)$. Thus $h_{p}(z) \leq \log (\|z\| / r)$ on $D$.

For $\xi$ fixed in $S(O, 1)$, by using again Montel's theorem, we prove property (iii). Let us prove the inequality (3) and the pointwise convergence on $D$ of the sequence $\left(h_{p}\right)$. For each function $f \in \mathcal{E}_{p}(D)$ and $g \in \mathcal{E}_{q}(D)$, the function $h=f g$ is in $\mathcal{E}_{p+q}(D)$. Consequently, $(p+q) h_{p+q} \geq \sup \left\{\log |f g|: f \in \mathcal{E}_{p}(D)\right.$, $\left.g \in \mathcal{E}_{q}(D)\right\}=p h_{p}+q h_{q}$. Let $z$ be fixed in $D \backslash\{O\}$ (for $z=O, h_{p}(O)=-\infty$ $\forall p \geq 1$ and then $\left.\lim _{p} h_{p}(O)=\sup _{p} h_{p}(O)=-\infty\right)$. Denote $L(z)=\lim \sup _{p} h_{p}(z)$ and $\ell(z)=\liminf _{p} h_{p}(z) . \forall \varepsilon>0, \exists p \geq 1$ such that $h_{p}(z) \geq L(z)-\varepsilon . \forall q \geq p$, $q=\alpha p+s$ with $\alpha \geq 1$ and $0 \leq s \leq p-1$. Then $q h_{q}(z)=(\alpha p+s) h_{\alpha p+s}(z) \geq$ $\alpha p h_{\alpha p}(z)+s h_{s}(z) \geq \alpha p h_{p}(z)+s h_{s}(z) \geq \alpha p(L(z)-\varepsilon)+s h_{s}(z)$ and we obtain that $\ell(z)=L(z)$. The inequality (4) follows from the inequality (3) and from the property (iii). The convergence of the sequence $\left(\gamma_{p}(D, \xi)\right)$ is proved as the convergence of $\left(h_{p}\right)$. $\left(\mathbf{N}^{*}\right)^{2}$,

Remark 3.2. From Lemma 3.1, we deduce two inequalities: $\forall(p, \alpha) \in$

$$
\begin{aligned}
& h_{\alpha p} \geq h_{p} \quad \text { on } D, \\
& \gamma_{\alpha p}(D, \xi) \leq \gamma_{p}(D, \xi), \quad \forall \xi \in S(O, 1) .
\end{aligned}
$$

In the following property, we give a first comparison of functions $h_{p}$ and $g_{D}$ and of capacities $\gamma_{p}(D, \xi)$ and $C_{D}(\xi)$.

Lemma 3.3. If $D$ is a bounded hyperconvex domain in $\mathbf{C}^{n}$ containing $O$, then for every $p \in \mathbf{N}^{*}$ :

$$
\begin{aligned}
& h_{p} \leq g_{D}, \quad \text { on } D, \text { and } \\
& \gamma_{p}(D, \xi) \geq C_{D}(\xi), \quad \text { for each } \xi \in S(O, 1) .
\end{aligned}
$$

Proof. For every function $f \in \mathcal{E}_{p}(D), \log |f| \in \operatorname{PSH}(D)$ and is negative on $D$. According to Lemma 3.1, we have $(\log |f(z)|) / p \leq \log \|z\|-\log r$ on $D$, if $B(O, r) \subset D$. Therefore, from the maximality of $g_{D}$, we obtain the inequality $(\log |f(z)|) / p \leq g_{D}(z) \forall z \in D$, which gives us (5). The inequality (6) is a consequence of (5) and of Lemma 3.1 (iii). 
Proposition 3.4. Let $D$ be a bounded strictly convex open set in $\mathbf{C}^{n}$, with analytic boundary and containing $O$. Then for all $p \in \mathbf{N}^{*}$ we have

$$
\begin{array}{cl}
g_{D}(z)=h_{p}, & \forall z \in D, \\
\gamma_{p}(D, \xi)=C_{D}(\xi), & \forall \xi \in S(O, 1) .
\end{array}
$$

Proof. Identity of functions $g_{D}$ and $h_{1}$ was proved by L. Lempert in [10]. We deduce the equality between $C_{D}(\xi)$ and $\gamma_{1}(D, \xi), \forall \xi \in S(O, 1)$. According to the inequalities $\left(3^{\prime}\right),\left(4^{\prime}\right),(5)$ and $(6)$, we get $\gamma_{p}(D, \xi)=C_{D}(\xi)$ on $S(O, 1)$ and $h_{p}=g_{D}$ on $D, \forall p \geq 1$.

Now, we are going to prove the convergence of the sequence $\left(h_{p}\right)$ to $g_{D}$, using an idea of J. P. Demailly (see [4]). Let us first suppose that $D$ is a bounded hyperconvex domain in $\mathbf{C}^{n}$ containing $O$. For any $p \in \mathbf{N}^{*}$, let $\mathcal{H}_{D}\left(p g_{D}\right)=\{f \in$ $\left.H(D): \int_{D}|f(z)|^{2} e^{-2 p g_{D}(z)} d \mu(z)<\infty\right\}$ be the Hilbert space provided with the scalar product

$$
(f, g)_{2, p}=\int_{D} f(z) \overline{g(z)} e^{-2 p g_{D}(z)} d \mu(z)
$$

(the norm is denoted by $\|\cdot\|_{2, p}$ ) and let $g_{p}$ be the function defined on $D$ by $g_{p}(z)=\left(\log \left(\sum_{l}\left|\sigma_{\ell}(z)\right|^{2}\right)\right) /(2 p)$, where $\left(\sigma_{\ell}\right)$ is an orthonormal basis of $\mathcal{H}_{D}\left(p g_{D}\right)$.

Just by using the fact that $\log (\|z\| / R) \leq g_{D}(z) \leq \log (\|z\| / r)$ on $D$, if $B(O, r) \subset D \subset B(O, R)$, we prove the following lemma.

Lemma 3.5. Let $D$ be a bounded hyperconvex domain in $\mathbf{C}^{n}$ containing $O$.

(1) If $p \geq n$, every function $f$ in $\mathcal{H}_{D}\left(p g_{D}\right)$ satisfies $D^{(\nu)} f(O)=0 \forall \nu \in$ $\mathbf{N}^{n} /|\nu| \leq p-n$.

(2) If $p<n$, then for every $\nu$ there is a function $f$ in $\mathcal{H}_{D}\left(p g_{D}\right)$ such that $D^{(\nu)} f(O) \neq 0$.

Lemma 3.6. If $D$ is a bounded hyperconvex domain in $\mathbf{C}^{n}$ containing $O$, then $\forall p \in \mathbf{N}^{*}$

$$
g_{p}(z)=\sup _{f \in B(O, 1)_{2, p}} \frac{1}{p} \log |f(z)| \quad \text { on } D,
$$

where $B(O, 1)_{2, p}$ is the unit ball in $\mathcal{H}_{D}\left(p g_{D}\right)$. Moreover, $g_{p}$ is a continuous psh function on $D$.

Proof. $\forall w \in D$, let $\phi_{w}$ be the evaluation linear form $f \mapsto f(w)$ on $\mathcal{H}_{D}\left(p g_{D}\right)$. We verify that its norm $\left\|\phi_{w}\right\|$ is equal to $\left(\sum_{l}\left|\sigma_{\ell}(w)\right|^{2}\right)^{1 / 2}$. Since $g_{D}$ is negative on $D$, the $L^{2}$-topology in the space $\mathcal{H}_{D}\left(p g_{D}\right)$ is stronger than the topology of 
uniform convergence on every compact set in $D$. Thus the series $\sum_{l}\left|\sigma_{\ell}\right|^{2}$ converges uniformly on every compact set in $D$ and is real analytic and continuous on $D$. Hence $g_{p}$ is a continuous and psh function on $D$.

Proposition 3.7. Let $D$ be a bounded hyperconvex domain in $\mathbf{C}^{n}$ containing $O$. Then there exist a constant $c_{1}>0$, depending only on $n$ and the diameter of $D$ and a constant $c_{2}>0$, such that

$$
\begin{aligned}
& g_{p}(z) \geq g_{D}(z)-\frac{c_{1}}{p} \\
& g_{p}(z) \leq \sup _{w \in B(z, r)} g_{D}(w)+\frac{1}{p}\left(\log c_{2}-n \log r\right)
\end{aligned}
$$

for all $z$ in $D$ and all real $r>0$ such that $r<\operatorname{dist}(z, b D)$. In particular, the sequence $\left(g_{p}\right)$ converges pointwise to the function $g_{D}$ on $D$.

Proof. We are going to use the following result of T. Ohsawa (Corollary 2 in [15]):

Let $Y$ be a pure dimensional closed complex submanifold of $\mathbf{C}^{n}$, let $D$ be a bounded pseudoconvex domain in $\mathbf{C}^{n}$ and $\varphi$ a psh function on $D$. Then, for any holomorphic function $f$ on $D \cap Y$ with $\int_{D \cap Y}|f|^{2} e^{-\varphi} d \mu_{Y}<+\infty$, there exists holomorphic extension $F$ to $D$ such that $\int_{D}|F|^{2} e^{-\varphi} d \mu \leq A \int_{D \cap Y}|f|^{2} e^{-\varphi} d \mu_{Y}$. The constant $A$ depends on $Y$ and $\sup \{\|z\|: z \in D\}$, but does not depend either on $f$ or on $\varphi$.

This result applied to the 0 -dimensional subvariety $Y=\{z\}$ ( $z$ is any point in $D$ ) and to the function $\varphi=2 p g_{D}$ ( $p$ is any integer $\geq 1$ ) shows that for any $a \in \mathbf{C}$ there exists a function $f$ in $H(D)$ such that $f(z)=a$ and

$$
\int_{D} \frac{|f(w)|^{2} e^{-2 p g_{D}(w)}}{\left(1+\|w-z\|^{2}\right)^{n+1}} d \mu(w) \leq c_{n}|f(z)|^{2} e^{-2 p g_{D}(z)},
$$

where $c_{n}$ (see the proof of the Corollary 2 in [15]) is a constant which only depends on the dimension $n$. As $1+\|w-z\|^{2} \leq 1+\operatorname{diam}(D)^{2}, \forall(z, w) \in D^{2}$, we obtain:

$$
\int_{D}|f(w)|^{2} e^{-2 p g_{D}(w)} d \mu(w) \leq\left(\operatorname{diam}(D)^{2}+1\right)^{n+1} c_{n} e^{-2 p g_{D}(z)}|a|^{2} .
$$

If we choose $a \in \mathbf{C}$ such that the right-hand side of the previous inequality is equal to 1 , then letting $c_{1}=\left(\log c_{n}+(n+1) \log \left(1+\operatorname{diam}(D)^{2}\right)\right) / 2$ and according to Lemma 3.6, we get the inequality (7). 
Let us prove now inequality (8). Take $z$ and $r$ as above. Let $f$ be a function in $B(O, 1)_{2, p}$. The mean value inequality applied to the psh function $|f|^{2}$ implies

$$
\begin{aligned}
|f(z)|^{2} & \leq \frac{c_{2}^{2}}{r^{2 n}} \int_{B(z, r)}|f(w)|^{2} d \mu(w) \\
& \leq \frac{c_{2}^{2}}{r^{2 n}}\left(\int_{B(z, r)}|f(w)|^{2} e^{-2 p g_{D}(w)} d \mu(w)\right) C(z),
\end{aligned}
$$

where $C(z)=\exp \left(2 p \sup \left\{g_{D}(w): w \in B(z, r)\right\}\right)$ and $c_{2}=\sqrt{n ! / \pi^{n}}$. Using again Lemma 3.6, we are done.

Now we suppose that $D$ is a bounded strictly hyperconvex domain in $\mathbf{C}^{n}$ containing $O$.

Lemma 3.8. $\forall j \geq 1$ and $\forall p \geq 1$ we have the following inequality

$$
\frac{p+n-1}{p} g_{p+n-1, D_{j}}-\frac{\log \left(c_{2} \delta(j)^{-n}\right)}{p} \leq h_{p} \text { on } D,
$$

where

$$
\begin{gathered}
B(0,1)_{2, p+n-1, D_{j}}=\left\{f \in H\left(D_{j}\right): \int_{D_{j}}|f|^{2} e^{-2(p+n-1) g_{D_{j}}} d \mu \leq 1\right\}, \\
\delta(j)=\operatorname{dist}\left(\bar{D}, b D_{j}\right), \text { and } \\
g_{p+n-1, D_{j}}=\sup \left\{\frac{(\log |f|)}{(p+n-1)}: f \in B(0,1)_{2, p+n-1, D_{j}}\right\} .
\end{gathered}
$$

Proof. Let $f \in B(0,1)_{2, p+n-1, D_{j}}$. There exists $z \in b D$ such that $|f(z)|=$ $\sup \{|f(w)|: w \in \bar{D}\}$. By the mean value inequality applied to the psh function $|f|^{2}$ on the ball $B(z, \delta(j)) \subset D_{j}$, we get

$$
|f(z)|^{2} \leq \int_{B(z, \delta(j))}|f(w)|^{2} d \mu(w) c_{2}^{2} / \delta(j)^{2 n} .
$$

As $g_{D_{j}}$ is negative on $D_{j}$, we deduce that

$$
\begin{aligned}
|f(z)|^{2} & \leq \frac{c_{2}^{2}}{\delta(j)^{2 n}} \int_{B(z, \delta(j))}|f(w)|^{2} e^{-2(p+n-1) g_{D_{j}}(w)} d \mu(w) \\
& \leq \frac{c_{2}^{2}}{\delta(j)^{2 n}}\|f\|_{2, p+n-1, D_{j}}^{2}
\end{aligned}
$$


From Lemmas 3.5 and 3.6 and the inequality above, we obtain the required inequality.

Finally, letting $p$ and $j$ go to infinity in the inequalities (5) and (9) and by Proposition 3.7 , we derive that the sequence $\left(h_{p}\right)$ converges pointwise to $g_{D}$ on $D$.

Now let us prove the convergence of the sequence $\left(\gamma_{p}(D, \xi)\right)$ to $C_{D}(\xi)$. First, we remark that the sequence $\left(h_{p^{\alpha}}\right)_{\alpha \geq 1}$ increases $(\forall p>1)$, according to the inequality $\left(3^{\prime}\right)$ and converges pointwise on $D$ to the function $g_{D}$.

Let us study the sequence of the Monge-Ampère operators $\left(\left(d d^{c} h_{m}\right)^{n}\right)_{m>1}$ on $D$. By Property 3.1 (ii), $h_{m}(z)$ is equivalent to $\log \|z\|$ when $z$ tends to $O$. Thus, according to the Theorem 3.9 of [3], we deduce that $\left(d d^{c} h_{m}\right)^{n}(\{O\})=(2 \pi)^{n}$ $\forall m \geq 1$. Let us denote by $\Omega$ the open set $D \backslash\{O\}$. The increasing sequence $\left(h_{p^{\alpha}}\right)_{\alpha \geq 1}$ is contained in $\operatorname{PSH}(D) \cap L_{l o c}^{\infty}(\Omega)$ and converges pointwise on $\Omega$ to the function $g_{D} \in \operatorname{PSH}(D) \cap L_{\mathrm{loc}}^{\infty}(\Omega)$. From Theorem 7.4 of [1], we deduce that the sequence of the Radon measures $\left(\left(d d^{c} h_{p^{\alpha}}\right)^{n}\right)_{\alpha \geq 1}$ converges, in the weak sence of measures on $\Omega$, to the measure identiqual to zero, since $\left(d d^{c} g_{D}\right)^{n}=0$ on $\Omega$. So the sequence of the Radon measures $\left(\left(d d^{c} h_{p^{\alpha}}\right)^{n}\right)_{\alpha \geq 1}$ converges to the measure $(2 \pi)^{n} \delta_{0}=\left(d d^{c} g_{D}\right)^{n}$, on $D$.

By Theorem 6.6 of [2], we get that the sequence $\left(\gamma_{p^{\alpha}}(D, \xi)\right)_{\alpha \geq 1}$ decreases to $C_{D}(\xi)$ quasi-everywhere in $S(O, 1)$ (i.e., except on a polar set in $S(O, 1)$, regarded as $\left.\mathbf{C P} \mathbf{P}^{n-1}\right)$, since

$$
\lim _{\alpha \rightarrow+\infty} \int_{D} \log (1+\|z\|)\left(d d^{c} h_{p^{\alpha}}\right)^{n}=\int_{D} \log (1+\|z\|)\left(d d^{c} g_{D}\right)^{n}=0 .
$$

Finally, since the sequence $\left(\gamma_{m}(D, \xi)\right)_{m}$ converges $\forall \xi \in S(O, 1)$, we are done.

4. Comparison of capacities in $\mathbf{C}^{n}$. Let $K$ be a compact set in $\mathbf{C}^{n}$ and $V_{K}$ be the extremal function on $\mathbf{C}^{n}$ associated with $K$ ([17], [21]) and defined by

$$
V_{K}(z)=\sup \left\{v(z): v \in L\left(\mathbf{C}^{n}\right) \text { and }\left.v\right|_{K} \leq 0\right\},
$$

where $L\left(\mathbf{C}^{n}\right)=\left\{v \in \operatorname{PSH}\left(\mathbf{C}^{n}\right): v(z) \leq \log (1+\|z\|)+O_{v}(1)\right\}$. Denote by $g_{K}$ the semicontinuous regularization $V_{K}^{*}$, called the generalized Green function for the compact set $K([21])$. J. Siciak $([17])$ proved that the function $g_{K}$ belongs to the set $L\left(\mathbf{C}^{n}\right)$ if and only if $K$ is a non-pluripolar compact set. From now on, we will assume this. Then we define $C(K)$, the harmonic capacity associated with the compact set $K$, by

$$
-\log C(K)=\gamma(K)=\limsup _{\|z\| \rightarrow \infty}\left(g_{K}(z)-\log \|z\|\right),
$$

where $\gamma(K)$ is the Robin constant of the set $K$. 
Let $\alpha$ be a positive integer and $\xi$ be in $S(O, 1)$. Denote by $\mathcal{P}_{\alpha}$ the space of all holomorphic polynomials of degree less or equal to $\alpha$ in $\mathbf{C}^{n}$ and by $\mathcal{P}_{\alpha}^{*}(\xi)$ the subspace of all polynomials $p$ such that $p(z)=\sum_{|\sigma| \leq \alpha-1} c_{\sigma} z^{\sigma}+c_{\alpha}(z \cdot \xi)^{\alpha}$, where $c_{\sigma}\left(\sigma \in \mathbf{N}^{n}\right)$ and $c_{\alpha}$ are complex numbers. For all polynomial $p$ in $\mathcal{P}_{\alpha}, \hat{p}$ is its homogeneous part of degree $\alpha$. We define

$$
\begin{aligned}
\mathcal{P}_{\alpha, u} & =\left\{p \in \mathcal{P}_{\alpha}:\|\hat{p}\|_{S(O, 1)} \geq 1\right\} & M_{\alpha}(K) & =\inf _{p \in \mathcal{P}_{\alpha, u}}\|p\|_{K} \\
\mathcal{P}_{\alpha, u}(\xi) & =\left\{p \in \mathcal{P}_{\alpha}:|\hat{p}(\bar{\xi})| \geq 1\right\} & & m_{\alpha}(K, \xi)=\inf _{p \in \mathcal{P}_{\alpha, u}(\xi)}\|p\|_{K} \\
\mathcal{P}_{\alpha, u}^{*}(\xi) & =\left\{p \in \mathcal{P}_{\alpha}^{*}(\xi):\left|c_{\alpha}\right| \geq 1\right\} & m_{\alpha}^{*}(K, \xi) & =\inf _{p \in \mathcal{P}_{\alpha, u}^{*}(\xi)}\|p\|_{K}
\end{aligned}
$$

For any compact set $K$ in $\mathbf{C}^{n}$, the sequence $\left(M_{\alpha}(K)^{1 / \alpha}\right)$ converges to a constant $\tau(K)$, called the Tchébycheff constant of the compact set $K$ (see [21], [18] and [11]). In the same manner we first prove that, $\forall \xi \in S(O, 1)$ and $\forall\left(\alpha_{1}, \alpha_{2}\right) \in \mathbf{N}^{2}, m_{\alpha_{1}+\alpha_{2}}(K, \xi) \leq m_{\alpha_{1}}(K, \xi) m_{\alpha_{2}}(K, \xi)$ and $m_{\alpha_{1}+\alpha_{2}}^{*}(K, \xi) \leq$ $m_{\alpha_{1}}^{*}(K, \xi) m_{\alpha_{2}}^{*}(K, \xi)$. Then we deduce that the sequences $\left(m_{\alpha}(K, \xi)^{1 / \alpha}\right)$ and $\left(m_{\alpha}^{*}(K, \xi)^{1 / \alpha}\right)$ are convergent and we respectively denote by $\tau(K, \xi)$ and $\tau^{*}(K, \xi)$ their limit. $\tau(K, \xi)$ and $\tau^{*}(K, \xi)$ are $\xi$-directional Tchébycheff constants associated to $K$.

At first let us compare Tchébycheff constants with harmonic capacities of a compact set in $\mathbf{C}^{n}$. For this we need the following result, which has been proved by V. P. Zaharyuta in the case of a L-regular compact set (i.e. a compact set $K$ in $\mathbf{C}^{n}$ for which the function $V_{K}$ is continuous), and then by J. Siciak in the general case.

Proposition 4.1. ([22], [17], [18]) Let $K$ be a compact set in $\mathbf{C}^{n}$.

$$
\sup _{\alpha \geq 1}\left\{\sup \left\{\frac{1}{\alpha} \log |p(z)|: p \in \mathcal{P}_{\alpha},\|p\|_{K} \leq 1\right\}\right\}=V_{K}(z), \quad \text { on } \mathbf{C}^{n} .
$$

Proposition 4.2. Let $K$ be a compact set in $\mathbf{C}^{n}$.

(1) $C(K)=\tau(K)$.

(2) If $K$ is L-regular, $\tau(K, \xi)=C_{K}(\bar{\xi})$, on $S(O, 1)$. Moreover, we have $\tau(K, \xi) \leq \tau^{*}(K, \xi)$ on $S(O, 1)$.

Proof. The equality $C(K)=\tau(K)$ results from [16], [23] and [8]. Next, let us prove property (ii), by using properties of the orthogonal polynomials and Proposition 4.1.

Let us remark that $m_{\alpha}(K, \xi)^{-1}=\sup \left\{|\hat{p}(\bar{\xi})|: p \in \mathcal{P}_{\alpha},\|p\|_{K} \leq 1\right\}$. Since $(\log |p|) / \alpha \leq g_{K}$ on $\mathbf{C}^{n}$, for all $p \in \mathcal{P}_{\alpha}$ such that $\|p\|_{K} \leq 1$, we deduce that $\tau(K, \bar{\xi}) \geq C_{K}(\xi)$.

Moreover, there exists a sequence $\left(B_{k}\right)$ of orthonormal polynomials in the Hilbert space $L^{2}(K)$, which satisfies Theorem 2 in [23]. Then $\forall \varepsilon>0$, there exists 
a strictly increasing sequence $\left(k_{j}\right)_{j}$ of integers such that $\left|\hat{B}_{k_{j}}(\bar{\xi})\right| \geq e^{\left(\gamma_{K}(\bar{\xi})-\varepsilon\right) d_{j}}$ $\forall j$, where $d_{j}$ is the degree of the polynomial $B_{k_{j}}$. According to BernsteinMarkov's inequality, there exists a constant $c_{\varepsilon}>0$ such that $\left\|B_{k_{j}}\right\|_{K} \leq c_{\varepsilon}(1+\varepsilon)^{d_{j}}$ $\forall j$. If we note $P_{j}=c_{\varepsilon}^{-1}(1+\varepsilon)^{-d_{j}} B_{k_{j}}$, then $\left\|P_{j}\right\|_{K} \leq 1$. We deduce that $m_{d_{j}}(K, \xi)^{-1} \geq\left|\hat{P}_{j}(\bar{\xi})\right| \geq c_{\varepsilon}^{-1}(1+\varepsilon)^{-d_{j}} e^{\left(\gamma_{K}(\bar{\xi})-\varepsilon\right) d_{j}}$ and $m_{d_{j}}(K, \xi)^{1 / d_{j}} \leq c_{\varepsilon}^{1 / d_{j}}(1+$ $\varepsilon) e^{-\gamma_{K}(\bar{\xi})+\varepsilon}$. Finally, letting $j$ go to infinity and $\varepsilon$ go to 0 in the inequality above, we obtain that $\tau(K, \xi) \leq C_{K}(\bar{\xi})$.

Remark 4.3. We can prove that $C(K)=\inf \left\{C_{K}(\xi): \xi \in S(O, 1)\right\}$ and $\tau(K)=\inf \{\tau(K, \xi): \xi \in S(O, 1)\}$. Therefore we can derive (i) from (ii) in the previous proposition, in the $L$-regular case.

Let us recall some information about analytic functionals [12]. Let $K$ be a compact set in the $n$-dimensional complex projective space $\mathbf{C P}^{n}$. We denote by $K^{*}$ its dual open set in $\left(\mathbf{C P}^{n}\right)^{*}$, defined by $K^{*}=\left\{\tau \in\left(\mathbf{C P}^{n}\right)^{*}\right.$ : $\left.\langle z, \tau\rangle=\sum_{j=0}^{n} z_{j} \tau_{j} \neq 0, \forall z \in K\right\}$, where $\left[\begin{array}{llll}z_{0}: & \ldots & : z_{n}\end{array}\right]$ and $\left[\begin{array}{llll}\tau_{0} & \ldots & \ldots & \tau_{n}\end{array}\right]$ are the homogeneous coordinates of $z \in \mathbf{C P}^{n}$ and $\tau \in\left(\mathbf{C P}^{n}\right)^{*}$, respectively. Geometrically $K^{*}$ corresponds to the set of all complex hyperplanes in $\mathbf{C P}^{n}$ not intersecting $K$. A compact set $K$ in $\mathbf{C P}^{n}$ is called linearly convex if through every point $z$ of the complement of $K$, it passes at least one complex hyperplane not intersecting $K$.

If $U$ is an open set in $\mathbf{C P}^{n}$, we denote by $H(U)$ the space of holomorphic functions in $U$, homogeneous of degree 0 . It is a Fréchet space endowed with the topology of the uniform convergence on every compact set in $U$. If $K$ is a compact set in $\mathbf{C P}^{n}$, we note $H(K)$ the space of holomorphic functions in an open neighbourhood of $K$. The topology on $H(K)$ is defined by the inductive limit $H(K)=\lim _{\longrightarrow} H(U)$, where $U$ is any open neighbourhood of $K$. We denote by $H^{\prime}(K)$ (respectively $H^{\prime}(U)$ ) the topological dual space of $H(K)$ (respectively $H(U))$. An element in $H^{\prime}(K)$ is called an analytic functional on $K$.

Let $K$ be a compact set in $\mathbf{C P}^{n}$ non-intersecting the hyperplane at infinity $\left\{z \in \mathbf{C P}^{n}: z_{0}=0\right\}$. We define the Fantappié transform $\mathcal{F} \mu$ of an analytic functional $\mu \in H^{\prime}(K)$, by

$$
\mathcal{F} \mu(\tau)=\mu_{z}\left(\tau_{0} z_{0} /\langle z, \tau\rangle\right) \quad \forall \tau \in K^{*} .
$$

This function is analytic on $K^{*}$ and is equal to zero on the hyperplane at infinity in $\left(\mathbf{C P}^{n}\right)^{*}\left\{\tau \in\left(\mathbf{C P}^{n}\right)^{*}: \tau_{0}=0\right\}$. The mapping $\mathcal{F}: H^{\prime}(K) \rightarrow H_{0}\left(K^{*}\right)$, where $H_{0}\left(K^{*}\right)$ is the closed subspace of $H\left(K^{*}\right)$ consisting of the functions equal to zero on the hyperplane at infinity, is linear and continuous.

Let $K$ be a compact set in $\mathbf{C}^{n}$. We define the Fourier-Laplace transform $\hat{\mu}$ of an analytic functional $\mu \in H^{\prime}(K)$ by

$$
\hat{\mu}(p)=\mu\left(e^{i p \cdot z}\right) \quad \forall p \in \mathbf{C}^{n} .
$$


( $\hat{\mu}$ is an entire function.)

In what follows, we will consider only compact sets $K$ in $\mathbf{C P}^{n}$ containing the point $P_{0}=[1: 0: \ldots: 0]$ and included in $\mathbf{C P}^{n} \backslash\left\{z_{0}=0\right\} \sim \mathbf{C}^{n}$. Therefore, $K^{*}$ is included in $\left(\mathbf{C P}^{n}\right)^{*} \backslash\left\{\tau_{0}=0\right\} \sim \mathbf{C}^{n *}$ and contains the point $P_{0}^{*}=$ $[1: 0: \ldots: 0]$. We write $K^{*}=\left\{\tau \in \mathbf{C}^{n *}: 1+\tau \cdot z \neq 0, \forall z \in K\right\}$, where $\tau \cdot z=\sum_{j=1}^{n} \tau_{j} z_{j}$. Under these conditions, the Fantappié transform of an analytic functional $\mu$ defined on $K$, is the function $\mathcal{F} \mu$ of $n$ complex variables defined by $\mathcal{F} \mu\left(\tau_{1}, \ldots, \tau_{n}\right)=\mu_{z}\left((1+\tau \cdot z)^{-1}\right)$.

Definition 4.4. Let $K$ be a linearly convex compact set in $\mathbf{C P}^{n}$ non-intersecting the hyperplane at infinity. We say that $K$ is a Martineau compact if the Fantappié transform $\mathcal{F}$ is an isomorphism from $H^{\prime}(K)$ to $H_{0}\left(K^{*}\right)$.

Martineau's main result in [12] is: Each convex compact set in $\mathbf{C}^{n}$ is a Martineau compact. For $n=1$, all compact sets in $\mathbf{C}$ are Martineau compacta, according to C. L. da Silva, A. Grothendieck and G. Köthe.

Definition 4.5. A linearly convex compact set $K$ in $\mathbf{C}^{n}$ is a regular Martineau compact if it has a bounded hyperconvex dual domain $K^{*}$ in $\mathbf{C}^{n *}$ and if it satisfies the following property: there exists an increasing sequence $\left(K_{j}\right)$ of Martineau compacta in $\mathbf{C}^{n}$ such that $\bigcup_{j} K_{j}=\stackrel{\circ}{K}$. In addition, we assume that $\left(K_{j}^{*}\right)$ is a decreasing sequence of bounded hyperconvex domains in $\mathbf{C}^{n *}$, satisfying properties (1) and (2), with $\bar{K}^{*}=\bigcap_{j} K_{j}^{*}$.

Now let us compare a Tchébycheff constant of a compact set and the harmonic capacity of its dual domain. For this, we need to introduce a new analytic capacity associated to the dual domain. Let $K$ be a compact set in $\mathbf{C}^{n}$ containing $O$. If $\mu$ is an analytic functional on $K$, then we note $\|\mu\|_{0}=\sup \{|\mu(h)|: h \in$ $\left.H(K),\|h\|_{K} \leq 1\right\}\left(\|\mu\|_{0}\right.$ can be infinite). Moreover, we verify that $\forall \tau \in K^{*}$ and $\forall \sigma \in \mathbf{N}^{n}, D^{(\sigma)} \mathcal{F} \mu(\tau)=(-1)^{|\sigma|}|\sigma| ! \mu\left(z^{\sigma} /(1+z \cdot \tau)^{|\sigma|+1}\right)$. We deduce that $D^{(\sigma)} \mathcal{F} \mu(O)=(-1)^{|\sigma|}|\sigma| ! \mu\left(z^{\sigma}\right), \forall \sigma \in \mathbf{N}^{n}$ and $d_{\xi}^{(\alpha)}(\mathcal{F} \mu)(0) / \alpha !=(-1)^{\alpha} \mu\left((z \cdot \xi)^{\alpha}\right)$, $\forall \alpha \in \mathbf{N}$. Let us define for all integer $\alpha \geq 1$, the subspace $\mathcal{F}_{\alpha}(K)$ of $H^{\prime}(K)$ by $\mathcal{F}_{\alpha}(K)=\left\{\mu \in H^{\prime}(K):\|\mu\|_{0} \leq 1, \mu\left(z^{\nu}\right)=0 \forall \nu \in \mathbf{N}^{n} /|\nu| \leq \alpha-1\right\}$. We denote by $\tilde{\mathcal{E}}_{\alpha}\left(K^{*}\right)$ its image in $H\left(K^{*}\right)$ by the Fantappié transform $\mathcal{F}$. Then we define the function $\tilde{h}_{\alpha}$ on $K^{*}$ and the $\xi$-directional analytic capacity of order $\alpha \tilde{\gamma}_{\alpha}(D, \xi)$ on $S(O, 1)$ by:

$$
\begin{aligned}
\tilde{h}_{\alpha} & =\sup _{\mu \in \mathcal{F}_{\alpha}(K)} \frac{1}{\alpha} \log |\mathcal{F} \mu|, \\
\tilde{\gamma}_{\alpha}\left(K^{*}, \xi\right) & =\left\{\sup _{\mu \in \mathcal{F}_{\alpha}(K)}\left|\mu\left((\xi \cdot z)^{\alpha}\right)\right|^{1 / \alpha}\right\}^{-1} \\
& =\left\{\sup _{\mu \in \mathcal{F}_{\alpha}(K)}\left|\frac{1}{\alpha !} d_{\xi}^{(\alpha)}(\mathcal{F} \mu)(0)\right|^{1 / \alpha}\right\}^{-1} .
\end{aligned}
$$


Property 4.6. Let $K$ be a compact set in $\mathbf{C}^{n}$ containing $O$. For all $\xi \in$ $S(O, 1)$, the sequence of the $\xi$-directional analytic capacities $\left(\tilde{\gamma}_{\alpha}\left(K^{*}, \xi\right)\right)$ converges to $\tau^{*}(K, \xi)^{-1}$.

Proof. We are going to express $\tilde{\gamma}_{\alpha}\left(K^{*}, \xi\right)$ through $m_{\alpha}^{*}(K, \xi)$. Let $\mu \in \mathcal{F}_{\alpha}(K)$ and $Q$ be any polynomial in $\mathcal{P}_{\alpha}^{*}(\xi)$ such that $Q(z)=\sum_{|\nu| \leq \alpha-1} c_{\sigma} z^{\sigma}+c_{\alpha}(z \cdot \xi)^{\alpha}$. Since $\mu\left(z^{\sigma}\right)=0 \forall \sigma \in \mathbf{N}^{n} /|\sigma| \leq \alpha-1$, we get that $\mu(Q)=c_{\alpha} \mu\left((z \cdot \xi)^{\alpha}\right)$. Thus, $\sup \left\{|\mu(Q)|: Q \in \mathcal{P}_{\alpha}^{*}(\xi),\|Q\|_{K} \leq 1\right\}=\tilde{m}_{\alpha}^{*}(K, \xi)\left|\mu\left((z \cdot \xi)^{\alpha}\right)\right|$. We denote by $T$ the restriction of the analytic functional $\mu$ to the subspace $\mathcal{P}_{\alpha}^{*}(\xi)$. By HahnBanach's Theorem, there exists a continuous linear form $\mu^{\prime}$ on $H_{u}(K)$, the space of holomorphic functions on a neighbourhood of $K$ endowed with the topology of the uniform convergence on $K$, such that its restriction to $\mathcal{P}_{\alpha}^{*}(\xi)$ is $T$ and such that its norm $\left\|\mu^{\prime}\right\|_{0}$ is equal to $\tilde{m}_{\alpha}^{*}(K, \xi)\left|\mu\left((z \cdot \xi)^{\alpha}\right)\right|$. Since the topology of the uniform convergence on $K$ is coarser than the inductive topology in $H(K), \mu^{\prime}$ is an analytic functional on $K$.

We have $\mu(Q)=\mu^{\prime}(Q) \forall Q \in \mathcal{P}_{\alpha}^{*}(\xi)$, thus $\mu\left(z^{\sigma}\right)=\mu^{\prime}\left(z^{\sigma}\right)=0 \forall \sigma \in \mathbf{N}^{n} /|\sigma| \leq$ $\alpha-1$ and $\left|\mu\left((z \cdot \xi)^{\alpha}\right)\right|=\left|\mu^{\prime}\left((z \cdot \xi)^{\alpha}\right)\right|=\left\|\mu^{\prime}\right\|_{0} \tilde{m}_{\alpha}^{*}(K, \xi)^{-1}$. Consequently,

$$
\begin{aligned}
\sup \left\{\left|\mu^{\prime}\left((z \cdot \xi)^{\alpha}\right)\right|: \mu^{\prime} \in \mathcal{F}_{\alpha}(K)\right\} & =\sup \left\{\left|\mu\left((z \cdot \xi)^{\alpha}\right)\right|: \mu \in \mathcal{F}_{\alpha}(K, \xi)\right\} \\
& =\tilde{m}_{\alpha}^{*}(K, \xi)^{-1}
\end{aligned}
$$

where $\mathcal{F}_{\alpha}(K, \xi)=\left\{\mu \in H^{\prime}(K), \mu\left(z^{\sigma}\right)=0 \forall \sigma \in \mathbf{N}^{n} /|\sigma| \leq \alpha-1, \sup \{|\mu(Q)|\right.$ : $\left.\left.Q \in \mathcal{P}_{\alpha}^{*}(\xi),\|Q\|_{K} \leq 1\right\} \leq 1\right\}$ and $\tilde{\gamma}_{\alpha}(K, \xi)=m_{\alpha}^{*}(K, \xi)^{-1 / \alpha}$.

In the next Proposition 4.7, we are going to compare the limit of this sequence $\left(\tilde{\gamma}_{\alpha}\left(K^{*}, \xi\right)\right)$ with the $\xi$-directional harmonic capacity $C_{K^{*}}(\xi)$ of the domain $K^{*}$, when $K^{*}$ is a bounded hyperconvex domain in $\mathbf{C}^{n *}$ containing $O$. Then, Theorem 2 will be a direct consequence of Propositions 4.2 and 4.7 and Property 4.6.

Proposition 4.7. Let $K$ be a compact set in $\mathbf{C}^{n}$ such that its dual open set $K^{*}$ is a bounded hyperconvex domain in $\mathbf{C}^{n *}$. Then, we have:

$$
\begin{aligned}
& \limsup _{\alpha \rightarrow \infty} \tilde{h}_{\alpha} \leq g_{K^{*}} \quad \text { on } K^{*}, \\
& \lim _{\alpha \rightarrow \infty} \tilde{\gamma}_{\alpha}\left(K^{*}, \xi\right) \geq C_{K^{*}}(\xi) \quad \forall \xi \in S(O, 1) .
\end{aligned}
$$

(ii) Moreover if $K$ is a regular Martineau compact, we get

$$
\begin{aligned}
\lim _{\alpha \rightarrow \infty} \tilde{h}_{\alpha} & =g_{K^{*}}, \quad \text { on } K^{*}, \\
\lim _{\alpha \rightarrow \infty} \tilde{\gamma}_{\alpha}\left(K^{*}, \xi\right) & =C_{K^{*}}(\xi), \quad \text { quasi-everywhere in } S(O, 1) .
\end{aligned}
$$


Proof. First let us note for any $\delta>0, K_{\delta}^{*}=\left\{p \in K^{*}: g_{K^{*}}(p)<-\delta\right\}$ and $K_{\delta}=\left(K_{\delta}^{*}\right)^{*}$. $K_{\delta}^{*}$ is a hyperconvex domain in $\mathbf{C}^{n *}$ containing $O$, relatively compact in $K^{*}$ and with pluricomplex Green function $g_{K^{*}}+\delta$ with logarithmic pole at $O . K_{\delta}$ is compact in $\mathbf{C}^{n}$ and contains $K$. For $\delta=0, K_{0}^{*}=K^{*}$ and $K_{0}=K$, if $K$ is linearly convex. In addition, we have a family of semi-norms defined on $H^{\prime}(K)$ by $\|\mu\|_{\delta}=\sup \left\{|\mu(h)|: h \in H\left(K_{\delta}\right),\|h\|_{K_{\delta}} \leq 1\right\}$, where $\delta$ is a nonnegative real number and $\|h\|_{K_{\delta}}=\sup \left\{|h(z)|: z \in K_{\delta}\right\}$. For $\varepsilon$ and $\delta \in \mathbf{R}$ such that $0 \leq \varepsilon<\delta$, let $\operatorname{dist}\left(K_{\varepsilon}, K_{\delta}^{*}\right)=\inf \left\{|1+z \cdot p|: z \in K_{\varepsilon}, p \in K_{\delta}^{*}\right\}$, which is $>0$.

Let $K$ be a compact set as in (i). By the definition of the Fantappié transform $\mathcal{F}$, we have for any $\mu \in H^{\prime}(K),|\mathcal{F} \mu(p)| \leq\|\mu\|_{\varepsilon} / \operatorname{dist}\left(K_{\varepsilon}, K_{\delta}^{*}\right)$ on $K_{\delta}^{*}$, $\forall(\varepsilon, \delta) \in \mathbf{R}^{2} / O \leq \varepsilon<\delta$. Thus $\tilde{\mathcal{E}}_{\alpha}\left(K^{*}\right) \subset\left(\operatorname{dist}\left(K_{\varepsilon}, K_{\delta}^{*}\right)\right)^{-1} \mathcal{E}_{\alpha}\left(K_{\delta}^{*}\right)$ and, due to the inequality (5), we get

$$
\tilde{h}_{\alpha} \leq-\frac{1}{\alpha} \log \left(\operatorname{dist}\left(K, K_{\delta}^{*}\right)\right)+g_{K^{*}}+\delta, \quad \text { on } K_{\delta}^{*} .
$$

Consequently, letting $\alpha$ go to infinity and $\delta$ go to 0 , we get $\lim \sup _{\alpha} \tilde{h}_{\alpha} \leq$ $g_{K^{*}}$ on $K^{*}$.

Since $\mathcal{F}_{\alpha}(K)$ is compact in $H^{\prime}(K)$ and $\mathcal{F}$ is continuous, $\tilde{\mathcal{E}}_{\alpha}\left(K^{*}\right)$ is a compact set in $H\left(K^{*}\right)$ and $\lim _{\lambda \rightarrow 0}\left(\tilde{h}_{\alpha}(\lambda \xi)-\log |\lambda|\right)=-\log \tilde{\gamma}_{\alpha}\left(K^{*}, \xi\right), \forall \xi \in S(O, 1)$. From inequality $(*)$, we deduce that

$$
\tilde{\gamma}_{\alpha}\left(K^{*}, \xi\right) \geq \operatorname{dist}\left(K, K_{\delta}^{*}\right)^{1 / \alpha} C_{K^{*}}(\xi) e^{-\delta}, \quad \forall \delta>0 .
$$

Now let us assume that the compact set $K$ is a regular Martineau compact. For all $j$, the Fantappié transform $\mathcal{F}$ is an isomorphism from $H^{\prime}\left(K_{j}\right)$ to $H\left(K_{j}^{*}\right)$. For all $\alpha \geq 1$, denote by $H_{\alpha}^{\prime}\left(K_{j}\right)$ the compact set in $H^{\prime}\left(K_{j}\right)$ defined by $H_{\alpha}^{\prime}\left(K_{j}\right)=$ $\mathcal{F}^{-1}\left(\mathcal{E}_{\alpha}\left(K_{j}^{*}\right)\right)\left(\mathcal{F}^{-1}\right.$ is continuous and $\mathcal{E}_{\alpha}\left(K_{j}^{*}\right)$ is compact in $\left.H\left(K_{j}^{*}\right)\right)$. Since $K_{j} \mathbf{b}$ $K$, there exists a constant $c_{\alpha}(j)>0$ such that $\sup _{\mu \in H_{\alpha}^{\prime}\left(K_{j}\right)}\|\mu\|_{0}=c_{\alpha}(j)<+\infty$ and $H_{\alpha}^{\prime}\left(K_{j}\right) \subset c_{\alpha}(j) \mathcal{F}_{\alpha}(K)$. In addition, $H_{\alpha+1}^{\prime}\left(K_{j}\right) \stackrel{\alpha}{\subset} H_{\alpha}^{\prime}\left(K_{j}\right)$ and $c_{\alpha+1}(j) \leq$ $c_{\alpha}(j)$; and, in the same way, $H_{\alpha}^{\prime}\left(K_{j}\right) \subset H_{\alpha}^{\prime}\left(K_{j+1}\right)$ and $c_{\alpha}(j) \leq c_{\alpha}(j+1)$. Since $\mathcal{F}$ is surjective, we have $\mathcal{E}_{\alpha}\left(K_{j}^{*}\right) \subset c_{\alpha}(j) \tilde{\mathcal{E}}_{\alpha}\left(K^{*}\right)$ and $h_{\alpha, j} \leq\left(\log c_{\alpha}(j)\right) / \alpha+\tilde{h}_{\alpha}$ on $K^{*}$, where the function $h_{\alpha, j}$ is equal to $\sup \left\{(\log |f|) / \alpha: f \in \mathcal{E}_{\alpha}\left(K_{j}^{*}\right)\right\}$. Since the sequence $\left(c_{\alpha}(j)\right)_{\alpha}$ decreases, we get

$$
h_{\alpha, j} \leq \frac{1}{\alpha} \log c_{1}(j)+\tilde{h}_{\alpha}, \quad \text { on } K^{*} .
$$

Letting $\alpha$ go to infinity and according to Theorem 1, we obtain that $g_{K_{j}^{*}} \leq$ $\liminf \tilde{h}_{\alpha}$ on $K^{*}$. Next, letting $j_{\tilde{h}}$ go to infinity and according to the property (1), we get that $g_{K^{*}} \leq \liminf _{\alpha} \tilde{h}_{\alpha}$ on $K^{*}$. Consequently, in view of (i) we 
derive that the sequence $\left(\tilde{h}_{\alpha}\right)$ converges pointwise to $g_{K^{*}}$ on $K^{*}$. Moreover, from inequality $(* *)$ we obtain

$$
\gamma_{\alpha}\left(K_{j}^{*}, \xi\right) \geq c_{1}(j)^{-1 / \alpha} \tilde{\gamma}_{\alpha}\left(K^{*}, \xi\right) .
$$

Since a countable union of pluripolar sets is again pluripolar [1], by Theorem 1 and by letting $\alpha$ next $j$ go to infinity, we get that $\lim _{\alpha} \tilde{\gamma}_{\alpha}\left(K^{*}, \xi\right) \leq C_{K^{*}}(\xi)$, quasi-everywhere in $S(O, 1)$. With property (i), we conclude.

Property 4.8. If $K$ is a closed polydisk in $\mathbf{C}^{n}$ with center at $O$, then there exists $\xi$ in $S(O, 1)$ such that $C_{K^{*}}(\xi)<C_{K}(\bar{\xi})^{-1}$.

Proof. Let $K=\bar{P}(O, R)$ in $\mathbf{C}^{n}$. Since $K$ is convex, it is a Martineau compact. $K$ is also $L$-regular; its generalized Green function $g_{K}$ and its $\xi$ directional harmonic capacity $C_{K}(\xi)$ are defined by

$$
\begin{aligned}
g_{K}(z) & =\sup _{1 \leq k \leq n} \log ^{+}\left(\left|z_{k}\right| / R_{k}\right) \quad \text { on } \mathbf{C}^{n}, \\
C_{K}(\xi) & =\left(\sup _{1 \leq k \leq n}\left(\left|\xi_{k}\right| / R_{k}\right)\right)^{-1} \quad \forall \xi \in S(O, 1)
\end{aligned}
$$

$\stackrel{\circ}{K}=\bigcup_{j \geq 1} \bar{P}\left(O, R^{(j)}\right)$, where $R^{(j)}=\left(R_{1}-1 / j, \ldots, R_{n}-1 / j\right)$. For all $j$ sufficiently large, $K_{j}=\bar{P}\left(O, R^{(j)}\right)$ is a Martineau compact, since it is convex as $K$.

Let us denote by $H$ and $H_{j}$ the functions defined on $\mathbf{C}^{n *}$ by $H(\tau)=\sum_{k=1}^{n} R_{k}\left|\tau_{k}\right|$ and $H_{j}(\tau)=\sum_{k=1}^{n}\left(R_{k}-1 / j\right)\left|\tau_{k}\right|$. Then the dual open sets $K^{*}$ and $K_{j}^{*}$ are defined by $K^{*}=\left\{\tau \in \mathbf{C}^{n *}: H(\tau)<1\right\}$ and $K_{j}^{*}=\left\{\tau \in \mathbf{C}^{n *}: H_{j}(\tau)<1\right\}$. These bounded open sets are convex and thus hyperconvex. Their pluricomplex Green functions with logarithmic pole at $O$ are respectively defined by $g_{K^{*}}=\log H$ on $K^{*}$ and $g_{K_{j}^{*}}=\log H_{j}$ on $K_{j}^{*}$. The $\xi$-directional harmonic capacities $C_{K^{*}}(\xi)$ and $C_{K_{j}^{*}}(\xi)$ are respectively equal to $H(\xi)^{-1}$ and $H_{j}(\xi)^{-1} \forall \xi \in S(O, 1)$. Moreover, let us remark that properties (1) and (2) are satisfied by $\left(K_{j}^{*}\right)$ and $K^{*}$. Consequently, $K$ is a regular Martineau compact in $\mathbf{C}^{n}$.

Then we verify that for $\xi=(1 / \sqrt{3}, \sqrt{2 / 3}, 0, \ldots, 0)$ and $R_{1}$ and $R_{2}$ such that $R_{2}>\sqrt{2} R_{1}, C_{K^{*}}(\xi)=\sqrt{3} /\left(R_{1}+\sqrt{2} R_{2}\right)<C_{K}(\bar{\xi})^{-1}=1 /\left(\sqrt{3} R_{1}\right)$.

5. Polynomial approximations of the exponential function on a compact set in $\mathbf{C}^{n}$. First, let us estimate the Fourier-Laplace transform of an analytic functional. 
If $\mu$ is an analytic functional on a compact set $K$ in $\mathbf{C}^{n}$, then we denote by $c_{\sigma}(\mu)$ the complex numbers $\mu\left(z^{\sigma}\right)\left(\sigma \in \mathbf{N}^{n}\right)$, called momenta of $\mu$. In addition, we use the same notations as in Section 4 (see the beginning of the proof of Proposition 4.7). The following proposition generalizes the theorem of G. Henkin and A. Shananin [5] and Proposition 1 of [13] in the case $n=1$.

Proposition 5.1. Let $K$ be a compact set in $\mathbf{C}^{n}$.

(1) Suppose that its dual open set $K^{*}$ is a bounded hyperconvex domain in $\mathbf{C}^{n *}$. Then $\forall N \in \mathbf{N}^{*}, \forall \mu \in H^{\prime}(K) / c_{\sigma}(\mu)=0$ if $|\sigma| \leq N, \forall \xi \in S(O, 1)$, $\forall \lambda \in \mathbf{C}, \forall(\tau, \delta) \in \mathbf{R}^{2}$ with $0 \leq \tau<\delta$ such that $\lambda \xi e^{i \theta} /(N+1) \in K_{\delta}^{*}$ $\forall \theta \in \mathbf{R}$ and $|z \cdot \lambda \xi|<N+1 \forall z \in K$, we have

$$
|\hat{\mu}(\lambda \xi)| \leq \frac{\|\mu\|_{\tau}}{\operatorname{dist}\left(K_{\tau}, K_{\delta}^{*}\right)}\left\{\frac{e^{\delta+1}|\lambda|\left(1+\varphi_{\xi}^{\prime}(\lambda /(N+1))\right)}{(N+1) C_{K^{*}}(\xi)}\right\}^{(N+1)}
$$

The function $\varphi_{\xi}^{\prime}$, which arises in the previous inequality, depends only on the compact set $K$ and $\xi$.

(2) Suppose now that the compact set $K$ is a Martineau compact. Then its dual open set $K^{*}$ is connected and we have, $\forall \xi \in S(O, 1), \forall \lambda \in \mathbf{C}, \forall N \in \mathbf{N}$, $\forall \alpha \in \mathbf{N}^{*}$ with $N=\alpha N^{\prime}+i$, where $i \in\{0, \ldots, \alpha-1\}$ and $N^{\prime} \in \mathbf{N}$ such that $\lambda \xi e^{i \theta} /(N+1) \in K^{*} \forall \theta \in \mathbf{R}$, there exists an analytic functional $\mu \in H^{\prime}(K)$ such that $c_{\sigma}(\mu)=0$ for all multi-index $\sigma /|\sigma| \leq N$ and

$$
|\hat{\mu}(\lambda \xi)| \geq \frac{|\lambda|^{N+1} \cdot e^{-\left|d_{\alpha} \lambda\right| / \alpha}}{(N+1) ! \gamma_{\alpha}\left(K^{*}, \xi\right)^{\alpha N^{\prime}} \cdot \gamma_{i+1}\left(K^{*}, \xi\right)^{i+1}}\left(1-O\left(\frac{|\lambda|^{2}}{\sqrt{N+1}}\right)\right) .
$$

The absolute value of $O\left(|\lambda|^{2} / \sqrt{N+1}\right)$ in the previous inequality is bounded above by $C|\lambda|^{2} / \sqrt{N+1}$, where $C$ is a constant depending only on the compact set $K$, the integers $\alpha, i$, and $\xi$.

Proof. Part A of this proposition is similar to Part A of the theorem in [5]. The goal is to estimate $|\mathcal{F} \mu|$ and next to estimate $|\hat{\mu}|$, by using an integral formula for $\hat{\mu}$. Part B of this proposition is inspired by Part B of the theorem in [5], but instead of using the $\xi$-directional harmonic capacity of the dual open set $K^{*}$, we introduce the $\xi$-directional analytic capacities of the same open set.

There exists a function $F_{\alpha} \in \mathcal{E}_{\alpha}\left(K^{*}\right)$ and a function $F_{i+1} \in \mathcal{E}_{i+1}\left(K^{*}\right)$ such that $d_{\xi}^{(\alpha)} F_{\alpha}(O) / \alpha !=\gamma_{\alpha}\left(K^{*}, \xi\right)^{-\alpha}$ and $d_{\xi}^{(i+1)} F_{i+1}(O) /(i+1) !=\gamma_{i+1}\left(K^{*}, \xi\right)^{-(i+1)}$. Then if we denote by $\Phi$ the function $\left(F_{\alpha}\right)^{N^{\prime}} F_{i+1}, \Phi \in \mathcal{E}_{N+1}\left(K^{*}\right)$ and

$$
d_{\xi}^{(N+1)} \Phi(0) /(N+1) !=\gamma_{\alpha}\left(K^{*}, \xi\right)^{-\alpha N^{\prime}} \gamma_{i+1}\left(K^{*}, \xi\right)^{-(i+1)} .
$$


Also, for any $\lambda$ and $\xi$ as in the statements and for $t \in \mathbf{C}$ such that $|t|=N+1$, we have

$$
\begin{aligned}
\Phi(\lambda \xi / t)=\gamma_{\alpha}\left(K^{*}, \xi\right)^{-\alpha N^{\prime}} \gamma_{i+1}\left(K^{*}, \xi\right)^{-(i+1)}\left(\frac{\lambda}{t}\right)^{N+1} \\
\cdot\left[\left(1+\frac{d_{\alpha} \lambda}{t}\right)^{N^{\prime}}\left(1+\frac{d_{i+1} \lambda}{t}\right)+O\left(\frac{|\lambda|^{2}}{N+1}\right)\right],
\end{aligned}
$$

where $O(|t|)$ with $t \in \mathbf{C}$, depends on $\alpha$ and $i$ but doesn't depend neither on $\lambda$, nor on $N$ sufficiently large. Since $K$ is a Martineau compact, there exists an unique analytic functional $\mu$ on $\mathrm{K}$ such that its Fantappié transform $\mathcal{F} \mu$ is $\Phi$. Since $\Phi \in \mathcal{E}_{N+1}\left(K^{*}\right), c_{\sigma}(\mu)=0 \forall \sigma \in \mathbf{N}^{n} /|\sigma| \leq N$.

We are going to use the following formula, which expresses $\hat{\mu}$ by means of $\mathcal{F} \mu$ for any analytic functional $\mu$ on $K$. Let $p \in \mathbf{C}^{n}$ and $R_{0}$ be a positive real number such that $p / t \in K^{*} \forall t \in S\left(R_{0}\right)=\left\{t \in \mathbf{C}:|t|=R_{0}\right\}$ and $|z \cdot p|<R_{0}$ $\forall z \in K$. We have:

$$
\hat{\mu}(p)=\frac{1}{2 i \pi} \int_{S\left(R_{0}\right)} \frac{e^{-i t}}{t} \cdot \mathcal{F} \mu(p / t) d t .
$$

We prove this formula with the help of Cauchy formula in one variable [5]. Then, applying it to $p=\lambda \xi$ and $R_{0}=N+1$, we obtain $\hat{\mu}(\lambda \xi)=J_{1}+J_{2}$, where

$$
\begin{gathered}
J_{1}=\frac{1}{2 i \pi} \int_{S(N+1)} \frac{e^{-i t}}{t} \gamma_{\alpha}\left(K^{*}, \xi\right)^{-\alpha N^{\prime}} \gamma_{i+1}\left(K^{*}, \xi\right)^{-i-1} \cdot\left(\frac{\lambda}{t}\right)^{N+1} \\
\cdot\left(1+d_{\alpha} \frac{\lambda}{t}\right)^{N^{\prime}}\left(1+d_{i+1} \frac{\lambda}{t}\right) d t \\
J_{2}=\frac{1}{2 i \pi} \int_{S(N+1)} \frac{e^{-i t}}{t} \gamma_{\alpha}\left(K^{*}, \xi\right)^{-\alpha N^{\prime}} \\
\cdot \gamma_{i+1}\left(K^{*}, \xi\right)^{-i-1}\left(\frac{\lambda}{t}\right)^{N+1} O\left(\frac{|\lambda|^{2}}{N+1}\right) d t .
\end{gathered}
$$

If we compute $J_{1}$ by the residus formula and note $P(\ell, N)=N^{\prime}\left(N^{\prime}-1\right) \ldots\left(N^{\prime}-\right.$ $\ell+1) /(N+\ell+1)(N+\ell) \ldots(N+2) \forall \ell \in\left\{1, \ldots, N^{\prime}\right\}$ and $P(0, N)=1$, we obtain

$$
\begin{array}{r}
\left|J_{1}\right|=\gamma_{\alpha}\left(K^{*}, \xi\right)^{-\alpha N^{\prime}} \cdot \gamma_{i+1}\left(K^{*}, \xi\right)^{-i-1} \frac{|\lambda|^{N+1}}{(N+1) !} \\
\left|\sum_{\ell=0}^{N^{\prime}} \frac{\left(-i d_{\alpha} \lambda\right)^{\ell}}{\ell !} P(l, N)\left(1-\frac{i d_{i+1} \lambda}{N+l+2}\right)\right| .
\end{array}
$$


The expression

$$
\Sigma=\left|\sum_{\ell=0}^{N^{\prime}} \frac{\left(-i d_{\alpha} \lambda\right)^{\ell}}{\ell !} P(\ell, N)\left(1-\frac{i d_{i+1} \lambda}{N+l+2}\right)\right|
$$

has a lower bound $[1-O(|\lambda| /(N+1))] e^{-\left|d_{\alpha} \lambda\right| / \alpha}$, where $|O(|t|)| \leq D|t|$ in a neighbourhood of 0 . The constant $D$ depends on $\alpha$ and i, but doesn't depend on $N$ sufficiently large. In addition $\left|J_{2}\right|$ satisfies the following inequality:

$$
\left|J_{2}\right| \leq \gamma_{\alpha}\left(K^{*}, \xi\right)^{-\alpha N^{\prime}} \gamma_{i+1}\left(K^{*}, \xi\right)^{-i-1}\left(\frac{e|\lambda|}{N+1}\right)^{N+1} O\left(\frac{|\lambda|^{2}}{N+1}\right) .
$$

Consequently, we get

$$
\begin{aligned}
|\hat{\mu}(\lambda \xi)| \geq & \left|J_{1}\right|-\left|J_{2}\right| \\
\geq & \gamma_{\alpha}\left(K^{*}, \xi\right)^{-\alpha N^{\prime}} \gamma_{i+1}\left(K^{*}, \xi\right)^{-i-1} \frac{|\lambda|^{N+1}}{(N+1) !} \\
& \quad\left[\left(1-O\left(\frac{|\lambda|}{N+1}\right)\right) e^{-\left|d_{\alpha} \lambda\right| / \alpha}-\frac{(N+1) !}{(N+1)^{N+1}} e^{N+1} O\left(\frac{|\lambda|^{2}}{N+1}\right)\right] .
\end{aligned}
$$

And according to Stirling's formula, we finally obtain inequality (11).

The following proposition, which is a consequence of Proposition 5.1, and Theorem 1 gives us Theorem 3 .

Proposition 5.2. Let $K$ be a compact set in $\mathbf{C}^{n}$.

(A) If $K^{*}$ is a bounded hyperconvex domain then we have for every $\xi \in S(O, 1)$, $\lambda \in \mathbf{C}$ and $\delta \geq 0$ :

$$
\limsup _{N \rightarrow+\infty} N \cdot E_{N}^{1 / N}\left(K_{\delta}, e^{\lambda \xi \cdot z}\right) \leq e^{\delta+1}|\lambda| C_{K^{*}}(\xi)^{-1}
$$

(B) If $K$ is a Martineau compact in $\mathbf{C}^{n}$, then we have for all compact set $K_{0}$ in $\mathbf{C}^{n}$ such that $K \subset \stackrel{\circ}{K_{0}}$, for every $p \in \mathbf{N}^{*}, \xi \in S(O, 1)$ and $\lambda \in \mathbf{C}$ :

$$
\liminf _{N \rightarrow+\infty} N \cdot E_{N}^{1 / N}\left(K_{0}, e^{\lambda \xi \cdot z}\right) \geq e|\lambda| \gamma_{p}\left(K^{*}, \xi\right)^{-1}
$$


Let us remark that for $n=1$, the first inequality results from [20] and the second one from [13].

Proof. First, let us prove Part A. Let $\delta \geq 0$ be fixed and $H_{u}\left(K_{\delta}\right)$ be the complex vector space of holomorphic functions on a neighbourhood of $K_{\delta}$, provided with the topology of uniform convergence on $K_{\delta}$. Let $\mathrm{f}$ be the entire function defined by $f(z)=e^{z \cdot \lambda \xi} . f \notin \mathcal{P}_{N}$ and $E_{N}\left(K_{\delta}, f\right)>0, \forall N \geq 0$. Let $\Phi_{1}$ be the continuous linear functional defined on the vector space $V\left(f, \mathcal{P}_{N}\right)$ (the subspace of $H_{u}\left(K_{\delta}\right)$ generated by the function $f$ and the subspace $\mathcal{P}_{N}$ ) by $\Phi_{1}(t f+p)=t, \forall p \in \mathcal{P}_{N}$ and $\forall t \in \mathbf{C}$. Then $\left\|\Phi_{1}\right\|_{V^{\prime}\left(f, \mathcal{P}_{N}\right)}=\sup \left\{\left|\Phi_{1}(g)\right|: g \in\right.$ $\left.V\left(f, \mathcal{P}_{N}\right),\|g\|_{K_{\delta}} \leq 1\right\}=\sup \left\{\left(\|p / t+f\|_{K_{\delta}}\right)^{-1}: p \in \mathcal{P}_{N}, p \neq 0, t \in \mathbf{C} \backslash\{0\}\right\}=$ $E_{N}\left(K_{\delta}, f\right)^{-1}$. By Hahn-Banach's theorem, this linear functional $\Phi_{1}$ can be extended as a continuous linear functional $\Phi$ on $H_{u}\left(K_{\delta}\right)$, such that $\|\Phi\|_{H_{u}^{\prime}\left(K_{\delta}\right)}=$ $\left\|\Phi_{1}\right\|_{V^{\prime}\left(f, \mathcal{P}_{N}\right)}$. Let $\mu$ be equal to $E_{N}\left(K_{\delta}, f\right) \Phi$. $\mu$ satisfies $\mu(f)=E_{N}\left(K_{\delta}, f\right)$, $\mu(p)=0 \forall p \in \mathcal{P}_{N}$ and $\|\mu\|_{H_{u}^{\prime}\left(K_{\delta}\right)}=\|\mu\|_{\delta}=1$. Since the topology of the uniform convergence on $K_{\delta}$ is coarser than the inductive topology on $H\left(K_{\delta}\right), \mu$ is an analytic functional on $K_{\delta}$. Let us remark that $\mathcal{F} \mu \in H\left(K_{\delta}^{*}\right)$.

By the same way as for the part A of Proposition 5.1, we prove that $\forall N \in \mathbf{N}^{*}$, $\forall \xi \in S(O, 1), \forall \lambda \in \mathbf{C}, \forall \varepsilon>0$ such that $\lambda \xi e^{i \theta} /(N+1) \in K_{\delta+\varepsilon}^{*} \forall \theta \in \mathbf{R}$ and $|z \cdot \lambda \xi|<N+1 \forall z \in K, \hat{\mu}$ satisfies

$$
|\hat{\mu}(-i \lambda \xi)| \leq \frac{\|\mu\|_{\delta}}{\operatorname{dist}\left(K_{\delta}, K_{\delta+\varepsilon}^{*}\right)}\left[\frac{e^{\delta+\varepsilon+1}|\lambda|\left(1+\varphi_{\xi}^{\prime}(\lambda /(N+1))\right)}{(N+1) C_{K^{*}}(\xi)}\right]^{N+1} .
$$

Since $\|\mu\|_{\delta}=1$ and $\hat{\mu}(-i \lambda \xi)=\mu_{z}\left(e^{z \cdot \lambda \xi}\right)=E_{N}\left(K_{\delta}, f\right)$, we deduce that

$$
\begin{gathered}
N \cdot E_{N}\left(K_{\delta}, f\right)^{1 / N} \leq \\
\frac{N(N+1)^{-(N+1) / N}}{\operatorname{dist}\left(K_{\delta}, K_{\delta+\varepsilon}^{*}\right)^{1 / N}}\left[e^{1+\delta+\varepsilon}|\lambda|\left(1+\varphi_{\xi}^{\prime}(\lambda /(N+1))\right) C_{K^{*}}(\xi)^{-1}\right]^{(N+1) / N} .
\end{gathered}
$$

Finally, letting $N$ go to infinity and $\varepsilon$ go to 0 in the previous inequality, we obtain (12).

Now let us prove Part $\mathrm{B}$ of this proposition. According to Proposition 5.1 B, we have : $\forall \xi \in S(O, 1), \forall \lambda \in \mathbf{C}, \forall N \in \mathbf{N}, \forall \alpha \in \mathbf{N}^{*}$ with $N=\alpha N^{\prime}+i$ where $i \in\{0, \ldots, \alpha-1\}$ and $N^{\prime} \in \mathbf{N}$ such that $\lambda \xi e^{i \theta} /(N+1) \in K^{*} \forall \theta \in \mathbf{R}$, there exists an analytic functional $\mu$ on $K$ such that $c_{\sigma}(\mu)=0$ for all $\sigma \in \mathbf{N}^{n} /|\sigma| \leq N$ and satisfying inequality (11). Let us note $\|\mu\|_{K_{0}}^{*}=\sup \left\{|\mu(h)|: h \in H\left(K_{0}\right)\right.$, $\left.\|h\|_{K_{0}} \leq 1\right\}$. Since $\hat{\mu}(-i \lambda \xi)=\mu_{z}\left(e^{z \cdot \lambda \xi}\right)=\mu_{z}\left(e^{z \cdot \lambda \xi}-p(z)\right) \forall p \in \mathcal{P}_{N}$, we deduce that $|\hat{\mu}(-i \lambda \xi)| \leq\|\mu\|_{K_{0}}^{*} \sup \left\{\left|e^{z \cdot \lambda \xi}-p(z)\right|: z \in K_{0}\right\}, \forall p \in \mathcal{P}_{N}$. Consequently, 
$E_{N}\left(K_{0}, f\right) \geq|\hat{\mu}(-i \lambda \xi)| /\|\mu\|_{K_{0}}^{*}$ and

$$
N \cdot E_{N}\left(K_{0}, f\right)^{1 / N} \geq \frac{N|\lambda|^{(N+1) / N}}{((N+1) !)^{1 / N}} \cdot\left[\frac{e^{-\left|d_{\alpha} \lambda\right| / \alpha}\left(1-O\left(|\lambda|^{2} / \sqrt{N+1}\right)\right)}{\|\mu\|_{K_{0}}^{*} \gamma_{\alpha}\left(K^{*}, \xi\right)^{\alpha N^{\prime}} \gamma_{i+1}\left(K^{*}, \xi\right)^{i+1}}\right]^{1 / N} .
$$

Letting $N$ go to infinity, we finally obtain the inequality (13).

\section{REFERENCES}

[1] E. Bedford and B. A. Taylor, A new capacity for plurisubharmonic functions. Acta Math. 149 (1982), 1-40.

[2] E. Bedford And B. A. TAYlor, Plurisubharmonic functions with logarithmic singularities. Ann. Inst. Fourier 38 (1988), 133-171.

[3] J. P. Demailly, Mesures de Monge-Ampere et mesures pluriharmoniques. Math. Z. 194 (1987), 519-564.

[4] J. P. Demailly, Regularization of closed positive currents and intersection theory. J. Alg. Geom. 1 (1992), 361-409.

[5] G. M. Henkin and A. Shananin, $\mathbf{C}^{n}$-capacity and multidimensional moment problem. Proc.Symp. on Value Dist. Theory in Sev. Compl. Var., ed. W. Stoll, Univ. of Notre Dame Press, Indiana 12 (1992), 69-85.

[6] M. Jarnicki and P. Pflug, Invariant Distances and Metrics in Complex Analysis. De Gruyter Expositions in Mathematics 9, 1993.

[7] M. KLimeK, Extremal psh functions and invariant pseudodistances. Bull. Soc. Math. France 113 (1985), 123-142.

[8] S. Kolodzies, The logarithmic capacity in $\mathbf{C}^{n}$. Ann. Pol. Math. 48 (1988), 253-267.

[9] P. LeLong, Notions capacitaires et fonctions de Green pluricomplexes dans les espaces de Banach. C. R. Acad. Sci. Paris 305 série J (1987), 71-76.

[10] L. Lempert, Solving the degenerate complex Monge-Ampère equation with one concentrated singularity. Math. Ann. 263 (1983), 515-532.

[11] N. Levenberg and B. A. Taylor, Comparison of capacities in $\mathbf{C}^{n}$. Lect. Notes in Math. 1094 (1984), 162-172.

[12] A. Martineau, Equations différentielles d'ordre infini. Bull. Soc. Math. France 95 (1967), 109-154.

[13] S. Nivoche, Caractérisation de la capacité harmonique en termes d'approximations polynomiales de la fonction exponentielle. C. R. Acad. Sci. Paris 315 Série I (1992), 1359-1364.

[14] S. Nivoche, Fonction de Green pluricomplexe, notions capacitaires et problèmes d'approximations dans $\mathbf{C}^{n}$. C. R. Acad. Sci. Paris 318 Série I (1994), 1087-1092.

[15] T. Ohsawa, On the extension of $L^{2}$ holomorphic functions II. Pub. Res. Inst. Math. Sci., Kyoto Univ. 24 (1988), 265-275.

[16] A. Sadullaev, Plurisubharmonic measures and capacities on complex manifolds. Russian Math. Surv. 36 (1981), 61-119.

[17] J. Siciak, Extremal plurisubharmonic functions in $\mathbf{C}^{n}$. Ann. Pol. Math 39 (1982), 175-211.

[18] J. SicIAK, Extremal plurisubharmonic functions and capacities in $\mathbf{C}^{n}$. Sophia Kokyuroku in Math. 14. Sophia Univ., Tokyo (1982).

[19] B. A. TAYLOR, An estimate for an extremal plurisubharmonic function on $\mathbf{C}^{n}$. Sém. P. Lelong, P. Dolbeault, H. Skoda (Analyse) 1982-83, Lect. Notes in Math., 318-328. 
[20] T. Winiarski, Approximation and interpolation of entire functions. Ann. Polon. Math. 23 (1970), 259-273.

[21] V. P. Zaharyuta, Transfinite diameter, Čebysev constant and capacity for compacta in $\mathbf{C}^{n}$. Math. of U.S.S.R. Sbornik 25 (1975), 350-364.

[22] V. P. ZahaRYuta, Extremal plurisubharmonic functions, orthogonal polynomials and Bernstein-Walsh theorem for analytic functions of several variables. Ann. Pol. Math. 33 (1976), 137-148 (in Russian).

[23] A. Zériahi, Capacité, constante de Čebysev et polynômes orthogonaux associés à un compact de $\mathbf{C}^{n}$. Bull. Soc. Math. $2^{\mathrm{e}}$ Série 109 (1985), 325-335.

Institut de Mathématiques (UMR 9994 du C.N.R.S.)

Equipe d'Analyse Complexe

Université Pierre et Marie Curie

Tour 46-00-5 $5^{\text {ème }}$ ét.-Boite 172

4, Place Jussieu-F-75252 Paris Cedex 05, France

Received: March 11th, 1994; revised: April 5th, 1995 